\title{
The effectiveness of ferric carboxymaltose in childbearing age with iron deficiency anemia
}

\author{
Alyami A ${ }^{1}$, Almohaya A , Alburaih D $\mathrm{D}^{1}$, Almurait $\mathrm{H}^{1}$, Elawad $\mathrm{M}^{2}$, AlHumaidi $\mathrm{A}^{2}$ and Alsuwaidan $\mathrm{S}^{3 *}$ \\ ${ }^{1}$ College of Pharmacy, Prince Norah Bint Abdulrahman University, Kingdom of Saudi Arabia \\ ${ }^{2}$ King Abdullah University Hospital, Prince Norah Bint Abdulrahman University \\ ${ }^{3}$ Research Consultant, Health Sciences Research Center, Prince Norah Bint Abdulrahman University, Kingdom of Saudi Arabia
}

\begin{abstract}
Iron deficiency is the most common minerals deficiency worldwide. Iron has a major role in producing hemoglobin in red blood cells, which is responsible for carrying oxygen to the body's tissues. Treatment for an iron inadequacy depends on the cause and severity of the condition. Intravenous iron preparations are highly recommended when anemic pregnant women unable to tolerate, respond accordingly with better adherence to the regimen.
\end{abstract}

Aim of the research: This study is conducted to evaluate the possibility of using ferric carboxymaltose (FCM) as a first line treatment in pregnant women with IDA; and the effect of other contributor factors such as age, education, occupation, number of pregnancies and most importantly is the time of receiving FCM in term of first, second and third trimesters.

Methodology: Participants were gone throughout personal interview answering some questions about their gestational situation and habits to achieve the study outcomes. All participants were pregnant women with abnormal hemoglobin whose either already received their first dose of FCM or those who are scheduled to take their first dose.

Results: Eighty-eight (88) pregnant women were participated in this study and their ages were divided into groups. The average of post FCM hemoglobin (10.99) after injection was significantly higher compared to the baseline hemoglobin (9.06). A shift to healthy and better response was shown after the FCM were shown in all participants.

Conclusion: A single intravenous injection of FCM improves the hemoglobin level in a significant consideration in anemic pregnant women. It showed also that the respond of severe anemic cases were more positive in improving the level of hemoglobin than mild and/or moderate cases. Blood transfusion could be avoided by using FCM injection to optimize the iron stores rapidly and effectively. Intravenous injection of FCM could be used as trend in the first and second trimester during pregnancy with or without IDA if the hemoglobin level is low. Moreover, intravenous iron can be given late in pregnancy in the third trimester when rapid restoration of the iron stores and hemoglobin is required to avoid blood transfusion at delivery.

\section{Introduction}

Anemia is a blood disorder in which hemoglobin (Hgb) concentration is less than the normal Hgb level. It is affected by age, sex, physiological condition(s) and altitude above the sea level of that person [1]. It is a global public health problem with major consequences affecting both developing and developed countries [2]. The most common type of anemia is iron deficiency anemia (IDA), which is defined as anemia because of insufficient iron [3]. Iron deficiency is the most common minerals deficiency worldwide. Women are at higher risk compared with men [4,5]. Iron has a major role in producing hemoglobin in red blood cells which is responsible for carrying oxygen to the body's tissues [6]. Iron is a mineral naturally found in food and supplements, and it is available in two dietary forms: heme like in (meat, poultry, and seafood) and non-heme in (ironfortified foods, plants, beans, nuts and vegetables). Essentially, meat and seafood are the richest sources of iron. However, some vegetables such as spinach, broccoli, and beetroot are considered as a good source for iron too. Although this is in disagreement with previous review concludes that "Vegetarians have a higher risk for developing low iron stores, iron depletion, and associated iron deficiency anemia, compared to non-vegetarians" [7].
According to world health organization (WHO) data, the prevalence of IDA in Saudi Arabia is $32.0 \%$ of pregnant women and $32.3 \%$ of women of reproductive age with anemia, which presents a moderate public health risk. Hemoglobin estimation is the most common and classical hematological screening test used for iron deficiency. According to (WHO), anemia is defined as low hemoglobin status; the cut off value for the pregnant women is $\mathrm{Hgb}<12 \mathrm{~g} / \mathrm{dl}$, mild anemia $(10.0-11.9 \mathrm{~g} / \mathrm{dl})$, moderate $(8.0-9.9 \mathrm{~g} / \mathrm{dl})$ and severe $(\leq 7.9 \mathrm{~g} / \mathrm{dl})$. However, bone marrow aspiration is the definitive test to assess iron stores and diagnose its deficiency. Serum ferritin is an alternative to the bone marrow aspiration, and it is the best test to distinguishing those with IDA from those who are not iron deficient $[2,8,9]$.

The level of serum ferritin, hemoglobin and haematocrit, and transferrin or total iron-binding capacity could evaluate iron

${ }^{\star}$ Correspondence to: Salem Alsuwaidan, Research Consultant, Health Sciences Research Center, Prince Norah Bint Abdulrahman University, Kingdom of Saudi Arabia, E-mail: alraces@hotmail.com

Key words: ferric carboxymaltose, iron deficiency, iron deficiency, hemoglobin

Received: August 26, 2019; Accepted: September 03, 2019; Published: September 09, 2019 
inadequacy. The symptoms of iron deficiency vary, depending on its severity. Iron deficiency anemia can cause chronic fatigue, hair loss, cold hands and feet, shortness of breath and many other symptoms in addition to several physical signs. One of the characteristics symptoms is pagophagia, which is known by a craving to eat substance without nutritional value such as ice, dust or paint due to iron deficiency $[10,11]$.

The reasons behind iron deficiency anemia during pregnancy can be classified into three causes. First, it can be as a result of the decrease in the hemoglobin level due to an increase in the maternal plasma volume. Second, begin the pregnancy with inadequate iron storage, which can lead to an inability to meet the requirements throughout the pregnancy. Last, an increase in the maternal demand for iron [12]. The body requirements for iron in pregnant woman nearly increases to 1000 $\mathrm{mg}$, which can be divided by, $350 \mathrm{mg}$ for placental and fetal growth, $250 \mathrm{mg}$ for blood loss at the delivery and the biggest part will be for the dilation in the red blood cell mass that will consume approximately $500 \mathrm{mg}$ iron [13]. Iron deficiency anemia lead to health problems such as rapid or irregular heartbeat, premature births and low birth weight babies and delayed growth and development [14].

Treatment for an iron inadequacy depends on the cause and severity of the condition. Iron deficiency anemia could be treated either orally or by intravenous (IV) injection. In spite of the fact that oral iron supplementations are considered as the first line option for the majority of pregnant women with IDA due to their effectiveness, safety, and lower cost; yet an intolerable side effects (gastrointestinal side effects), non-compliance and/or predisposing pathology such as malabsorption (celiac disease) may limit the use of oral ferrous supplements [15].

On the other hand, intravenous iron preparations are highly recommended when anemic pregnant women unable to tolerate, respond accordingly with better adherence to the regimen. Ferric carboxymaltose (FCM) is a new type of iron III complex, dextran free, which makes it possible to be administered without a test dose for hypersensitivity, has a neutral $\mathrm{PH}$ (5.0-7.0) and physiological osmolality. Therefore, up to $1000 \mathrm{mg}$ as single dose can be infused over 15-30 minutes, with lower side effects than oral iron supplement thus the patients are more compliant with injectable dosage form. If the patient did not responded adequately after a single dose of FCM, another dose can be administered one week later [10,16-18].

Ferrous as an element is a trend use for all pregnant women in most if not all gynecological clinics, usually the practitioners start with ferrous sulfate (FS) $325 \mathrm{mg}$ orally or ferrous gluconate (FG) $300 \mathrm{mg}$ orally. However, the starting point to prescribe ferrous is crucial to decide whether if the first, second or third trimester; moreover what is the most appropriate and effective way to initiate FCM injection during pregnancy; and what is the most cost effective treatment specifically if the pregnant women with IDA [19].

\section{Aim of the research}

This study is conducted to evaluate the possibility of using FCM as a first line treatment in pregnant women with IDA; and the effect of other contributor factors such as age, education, occupation, number of pregnancies and most importantly is the time of receiving FCM in term of first, second and third trimester.

\section{Methodology}

Study design: This is a cross-sectional, prospective study conducted to monitor symptoms improvement and normalization of hemoglobin levels of the recruited participants.
Subjects: This study was conducted with a sample of eightyeight (88) pregnant women aged between (20) and (44) years old; whom participated throughout personal interview answering some questions about their gestational situation and habits to achieve the study outcomes. All participants were pregnant women with abnormal hemoglobin whose either already received their first dose of ferric carboxymaltose or those who are scheduled to take their first dose. Women who are post-delivery or pregnant women with normal hemoglobin are excluded from the study.

Data analysis: Data were analyzed using SPSS 21.0 version statistical software. Descriptive statistics (mean, standard deviation, frequencies and percentage) were used to describe the quantitative and categorical variables. Student's paired- $t$ test was used to compare the mean values of quantitative variables $(\mathrm{Hgb})$ between the baseline and post ferric carboxymaltose (FCM). One-way analysis of variance was used to compare the mean difference of Hgb values in relation to the categorical variables, which has more than two categories. A p-value of $\leq 0.05$ was used to report the statistical significance of findings to report the precision of results.

Setting: Research data were obtained from King Abdullah University Hospital at Princess Noura bint Abdulrahman University.

Ethical consideration: All the participants involved in this study provided written informed consent acknowledging the investigation's purpose and were assured of the confidentiality of the results. Institutional review board approval for the research was obtained from Health Sciences Research Center at Princess Noura bint Abdulrahman University, with IRB Log Number: 18-1098.

\section{Results}

Eighty-eight (88) pregnant women were participated in this study and their ages were divided into groups, from 20-25 years were 13 participants (14.8\%), from 26-30 years were 28 (31.8\%), from 31 to 35 years were $27(30.7 \%)$, and for 36 years and older were $20(22.7 \%)$. Their educational level was distributed as following: under high school 2 (2.3\%), high school 32 (36.4\%), bachelor's degree 41 (46.6\%), and master's degree or above $13(14.8 \%)$. Their occupational status was assigned as employed 48 (54.5\%) and un-employed 40 (45.5\%). More details for the timing of FCM injection during the gestational trimester whether if it is first, second or third trimester, were shown in (Table 1 ), together with information related to the number of deliveries. The average of post ferric carboxymaltose hemoglobin (10.99) after injection was significantly higher compared to the baseline hemoglobin (9.06) $(\mathrm{P}<0.0001$; 95\% CI: 1.65-2.21).

In regard to age groups and its efficient in absorbing Hgb before FCM injection as a baseline and after FCM injection, compared to the whole group as shown in (Figure 1). The range of hemoglobin level between baseline and after FCM injection was (9.06-10.99), showing the response of the participants after FCM injection with a difference of 1.93 for the whole group, where the maximum difference was 2.16 found with the group of 25 years and lower with no significant difference $(p>0.05)$ compared with any of other age group including the minimal difference for the age group of 30-35 years with 1.7.

Another comparison for level of hemoglobin was found when FCM was injected within the gestational trimester, first, second and third trimester. It was found that although the third trimester with the highest hemoglobin level compare to the others, yet the first trimester showed the best response with a difference between the baseline and post FCM injection with 2.15. None of these differences were calculated as significantly different. Figure 2 showed relative differences between 
Table 1. Demographic data showing distribution of the study participants according to age group

\begin{tabular}{|c|c|c|c|c|c|c|}
\hline \multicolumn{2}{|c|}{ Age (year) } & \multirow{2}{*}{$\begin{array}{c}\text { 25yr \& low } \\
\mathrm{n}=13(14.8 \%)\end{array}$} & \multirow{2}{*}{$\begin{array}{c}26-30 y r \\
n=28(31.8 \%)\end{array}$} & \multirow{2}{*}{$\begin{array}{c}31-35 y r \\
n=27(30.7 \%)\end{array}$} & \multirow{2}{*}{$\begin{array}{l}36 y r \& \text { above } \\
n=20(22.7 \%)\end{array}$} & \multirow{2}{*}{$\begin{array}{c}\text { All subjects } \\
\mathbf{n}=\mathbf{8 8}(\mathbf{1 0 0} \%)\end{array}$} \\
\hline & No. Participants & & & & & \\
\hline & & Freq. (\%) & Freq. (\%) & Freq. (\%) & Freq. (\%) & Freq. (\%) \\
\hline \multirow{4}{*}{ 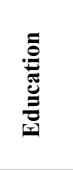 } & Under Hi. School & & $1(3.6 \%)$ & & $1(5 \%)$ & $2(2.3 \%)$ \\
\hline & High School & $10(76.9 \%)$ & $11(39.3 \%)$ & $6(22.2 \%)$ & $5(25 \%)$ & $32(36.4 \%)$ \\
\hline & Bachelor & $3(23.1 \%)$ & $15(53.6 \%)$ & $14(51.9 \%)$ & $9(45 \%)$ & $41(46.6 \%)$ \\
\hline & Master or Above & & $1(3.6 \%)$ & $7(25.9 \%)$ & $5(25 \%)$ & $13(14.8 \%)$ \\
\hline \multicolumn{2}{|c|}{ Employed } & $3(23.1 \%)$ & $11(39.3 \%)$ & $19(70.4 \%)$ & $15(75 \%)$ & $48(54.5 \%)$ \\
\hline \multicolumn{2}{|c|}{ Unemployed } & $10(76.9 \%)$ & $17(60.7 \%)$ & $8(29.6 \%)$ & $5(25 \%)$ & $40(45.5 \%)$ \\
\hline \multirow{3}{*}{ 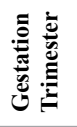 } & first & $6(46.2 \%)$ & $10(35.7 \%)$ & $14(51.9 \%)$ & $9(45 \%)$ & $39(44.3 \%)$ \\
\hline & Second & $3(23.1 \%)$ & $6(21.4 \%)$ & $6(22.2 \%)$ & $3(15 \%)$ & $18(20.5 \%)$ \\
\hline & Third & $4(30.8 \%)$ & $12(42.9 \%)$ & $7(25.9 \%)$ & $8(40 \%)$ & $31(35.2 \%)$ \\
\hline \multirow{4}{*}{ 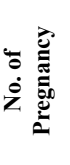 } & None & $8(61.5 \% 0$ & $11(39.3 \%)$ & $6(22.2 \%)$ & $1(5 \%)$ & $26(29.5 \%)$ \\
\hline & 2 times & $5(38.5 \%)$ & $6(21.4 \%)$ & $6(22.2 \%)$ & $2(10 \%)$ & $19(21.6 \%)$ \\
\hline & 3 times & & $6(21.4 \%)$ & $8(29.6 \%)$ & $5(25 \%)$ & $19(21.6 \%)$ \\
\hline & 4 and above & & $5(17.9 \%)$ & $7(25.9 \%)$ & $12(60 \%)$ & $24(27.2 \%)$ \\
\hline
\end{tabular}

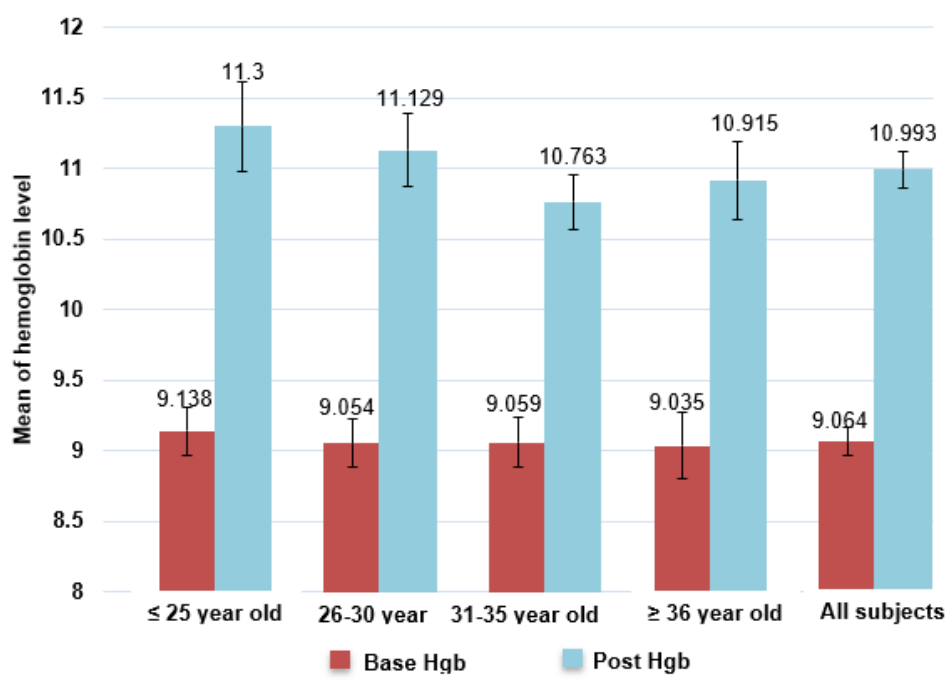

Figure 1. Comparison between basic mean for hemoglobin level and mean of hemoglobin after "FMC injection" according to age group

gestational trimesters first, second and third trimester compared with the total group before and after receiving one full dose of FCM.

Participants (at baseline) were classified in accordance with WHO classification to mild, moderate and severe anemia. A shift to healthy and better response was shown after the FCM with 14 participants were shown within healthy hemoglobin level, compared with none at the baseline. Then a big shift 8,70 and 10 out of the total participants as baseline before FCM injection for severe, moderate and mild anemia correspondingly. The improvement had shown after the FCM injection with only one participant with severe anemia or low hemoglobin level, 15 participants for moderate, and 58 participants become with mild anemia. It is also shown that 14 participants were getting full improvement and considered as healthy or non-anemic women. (Table 2) showed full details for all the data of this study mainly the shift of hemoglobin level during severe, moderate and mild to moderate, mild and healthy hemoglobin levels before and after receiving FCM.

\section{Discussion}

This is the first prospective study for ferric carboxymaltose in Saudi Arabia among pregnant women presenting with IDA.
An intravenous ferric carboxymaltose was remarkably increased hemoglobin levels without serious side effects were recorded. Only one case in this study had experienced dizziness, which were reported at the emergency department after FCM injection. FCM shown to be effective in childbearing age women with IDA, this phenomenon had been shown noticeable with those who aged with 25 or younger, where they have remarkably better response compared with other groups of age due to the ability to quickly absorb the iron as shown in (Figure 1). This difference is numerically deferent but not statistically deferent. Age as a factor in pregnant women with IDA had not been studied elsewhere; whereas in this study, age was divided into 4 age groups to study the associated differences of $\mathrm{Hgb}$ levels post FCM injection.

Results from this FCM analysis of pregnant women with IDA during first, second and third trimester of pregnancy showed that Hgb levels increased after FCM treatment with full safety parameters; therefore, intravenous FCM could be considered as a first line treatment for pregnant women with IDA in case of severe cases. This is in contrast to Centers for Disease Control and Prevention (CDC) [20], WHO [21], and Society of Obstetricians and Gynaecologists of Canada [22], where all these authorities recommended oral iron 
Table 2. Representing factors that affecting on hemoglobin level before and after receiving FCM

\begin{tabular}{|c|c|c|c|c|c|c|c|}
\hline & \multicolumn{3}{|c|}{ Basline IDA (before FCM) } & \multicolumn{4}{|c|}{ After FCM-Haemoglobin } \\
\hline & Sever & Moderate & Mild & Sever & Moderate & Mild & Healthy \\
\hline & $<7.9 \mathrm{gm} / \mathrm{dl}$ & $8-9.9 \mathrm{gm} / \mathrm{dl}$ & $10-11.9 \mathrm{gm} / \mathrm{dl}$ & $<7.9 \mathrm{gm} / \mathrm{dl}$ & $8-9.9 \mathrm{gm} / \mathrm{dl}$ & $10-11.9 \mathrm{gm} / \mathrm{dl}$ & $>11.9 \mathrm{gm} / \mathrm{dl}$ \\
\hline Uneducated & & $2(2.9 \%)$ & & 0 & 0 & 0 & $2(14.3 \%)$ \\
\hline Under H. School & $4(50 \%)$ & $27(38.6 \%)$ & $1(10 \%)$ & $1(100 \%)$ & $3(20 \%)$ & $23(39.7 \%)$ & $5(35.7 \%)$ \\
\hline High School & $2(25 \%)$ & $31(44.3 \%)$ & $8(80 \%)$ & 0 & $8(53.3 \%)$ & $27(46.6 \%)$ & $6(42.9 \%)$ \\
\hline Bachelor & $2(25 \%)$ & $10(14.3 \%)$ & $1(10 \%)$ & 0 & $4(26.7 \%)$ & $8(13.8 \%)$ & $1(7.1 \%)$ \\
\hline Employed & $5(62.5 \%)$ & $38(54.3 \%)$ & $5(50 \%)$ & & $10(66.7 \%)$ & $32(55.2 \%)$ & $6(42.9 \%)$ \\
\hline Unemployed & $3(37.5 \%)$ & $32(45.7 \%)$ & $5(50 \%)$ & & $5(33.3 \%)$ & $26(44.8 \%)$ & $8(57.1 \%)$ \\
\hline First tri & $6(75 \%)$ & $30(42.9 \%)$ & $3(30 \%)$ & & $6(40 \%)$ & $27(46.6 \%)$ & $6(42.9 \%)$ \\
\hline Second tri & $1(12.5 \%)$ & $14(20 \%)$ & $3(30 \%)$ & & $3(20 \%)$ & $14(24.1 \%)$ & 0 \\
\hline Third tri & $1(12.5 \%)$ & $26(37.1 \%)$ & $4(40 \%)$ & & $6(40 \%)$ & $17(29.3 \%)$ & $8(57.1 \%)$ \\
\hline 1st time Preg & $2(25 \%)$ & $19(27.1 \%)$ & $5(50 \%)$ & & $2(13.3 \%)$ & $18(31 \%)$ & $6(42.9 \%)$ \\
\hline 2nd time Preg & $1(12.5 \%)$ & $16(22.9 \%)$ & $2(20 \%)$ & & $3(20 \%)$ & $15(25.9 \%)$ & $1(7.1 \%)$ \\
\hline 3rd time Preg & $4(50 \%)$ & $15(21.4 \%)$ & 0 & & $5(33.3 \%)$ & $11(19 \%)$ & $2(14.3 \%)$ \\
\hline 4th and above & $1(12.5 \%)$ & $20(28.5 \%)$ & $3(30 \%)$ & & $5(33.3 \%)$ & $14(24.1 \%)$ & $5(35.6 \%)$ \\
\hline Vegetarian & 0 & $3(4.3 \%)$ & $1(10 \%)$ & & 0 & $4(6.9 \%)$ & 0 \\
\hline Non-veget & $8(100 \%)$ & $67(95.7 \%)$ & $9(90 \%)$ & $1(100 \%)$ & $15(100 \%)$ & $54(93.1 \%)$ & $14(100 \%)$ \\
\hline Diet w/ Fe & $3(37.5 \%)$ & $22(31.4 \%)$ & $4(40 \%)$ & & $4(26.7 \%)$ & $22(37.9 \%)$ & $3(21.4 \%)$ \\
\hline Diet w/No Fe & $5(62.5 \%)$ & $48(68.6 \%)$ & $6(60 \%)$ & $1(100 \%)$ & $11(73.3 \%)$ & $36(62.1 \%)$ & $11(78.6 \%)$ \\
\hline Craving & $2(25 \%)$ & $23(32.9 \%)$ & $3(30 \%)$ & & $3(20 \%)$ & $21(36.2 \%)$ & $4(28.6 \%)$ \\
\hline Sometimes & $6(75 \%)$ & $47(67.1 \%)$ & $7(70 \%)$ & $1(100 \%)$ & $12(80 \%)$ & $37(63.8 \%)$ & $10(71.4 \%)$ \\
\hline Sign_sym & $7(87.5 \%)$ & $55(78.6 \%)$ & $7(70 \%)$ & $1(100 \%)$ & $12(80 \%)$ & $48(82.8 \%)$ & $8(57.1 \%)$ \\
\hline Maybe & $1(12.5 \%)$ & $15(21.4 \%)$ & $3(30 \%)$ & & $3(20 \%)$ & $10(17.2 \%)$ & $6(42.8 \%)$ \\
\hline No Ferinject & $5(62.5 \%)$ & $43(61.4 \%)$ & $8(80 \%)$ & & $7(46.7 \%)$ & $41(70.7 \%)$ & $8(57.1 \%)$ \\
\hline Had Ferinject & $3(37.5 \%)$ & $27(38.6 \%)$ & $2(20 \%)$ & $1(100 \%)$ & $8(53.3 \%)$ & $17(29.3 \%)$ & $6(42.8 \%)$ \\
\hline Fe Suplmnt & $7(87.5 \%)$ & $59(84.3 \%)$ & $9(90 \%)$ & $1(100 \%)$ & $14(93.3 \%)$ & $47(81 \%)$ & $13(92.9 \%)$ \\
\hline No Fe Supp & $1(12.5 \%)$ & $11(15.7 \%)$ & $1(10 \%)$ & & $1(6.7 \%)$ & $11(19 \%)$ & $1(7.1 \%)$ \\
\hline 20-25yr Age & 0 & $13(18.6 \%)$ & 0 & & $2(13.3 \%)$ & $9(15.5 \%)$ & $2(14.3 \%)$ \\
\hline 26-30yr Age & $2(25 \%)$ & $22(31.4 \%)$ & $4(40 \%)$ & $1(100 \%)$ & $2(13.3 \%)$ & $19(32.8 \%)$ & $6(42.9 \%)$ \\
\hline 35-36yr Age & $4(50 \%)$ & $19(27.1 \%)$ & $4(40 \%)$ & & $5(33.3 \%)$ & $20(34.5 \%)$ & $2(14.3 \%)$ \\
\hline $37 \mathrm{yr}$ and more & $2(25 \%)$ & $16(22.9 \%)$ & $2(20 \%)$ & & $6(40 \%)$ & $10(17.2 \%)$ & $4(28.6 \%)$ \\
\hline Total; n (\%) & $8(100 \%)$ & $70(100 \%)$ & $10(100 \%)$ & $1(100 \%)$ & $15(100 \%)$ & $58(100 \%)$ & $14(100 \%)$ \\
\hline
\end{tabular}

Note: "Base IDA" have only mild, moderate and sever IDA, while after injection have only one case with sever IDA

supplementation in pregnant women as first line therapy. Intravenous iron is recommended when oral iron is poorly tolerated, absorption is likely to be impaired, the response to oral iron is inadequate, or when rapid restoration of $\mathrm{Hgb}$ and iron stores is required [23].

Oral iron supplementation could increase hemoglobin and ferritin levels in pregnancy, with or without IDA. This study demonstrated safe and effective use of FCM infusion in pregnant women during the first, second, and third trimester of pregnancy. Safety profile for intravenous FCM had been shown, having the practical advantage of allowing a higher iron dose in one time of administration (minimizing repeated administration times and increasing patient comfort) [24]. Another study showed Hgb levels significantly increased above baseline levels with $66 \%$ of women reported an improvement of their wellbeing with mostly minor and self-limiting side effects [25]. The cases described in this study was also in line with the retrospective case-control study from the Netherlands reporting similar significant increases in maternal Hgb levels above baseline and low rates of adverse outcomes [24]. Third trimester cases in this study had improvement with $21.3 \%$ with a single dose of intravenous injection of FCM (Figure 2).

It was noticed from this study that Ferric Carboxymaltose (FCM) was significantly increased hemoglobin levels, this increment in Hgb had been noticed started at 3 to 4 weeks interval post FCM dose; this achievement also had been previously studied [26]. The responses of severe anemic cases are more positive in improving the level of

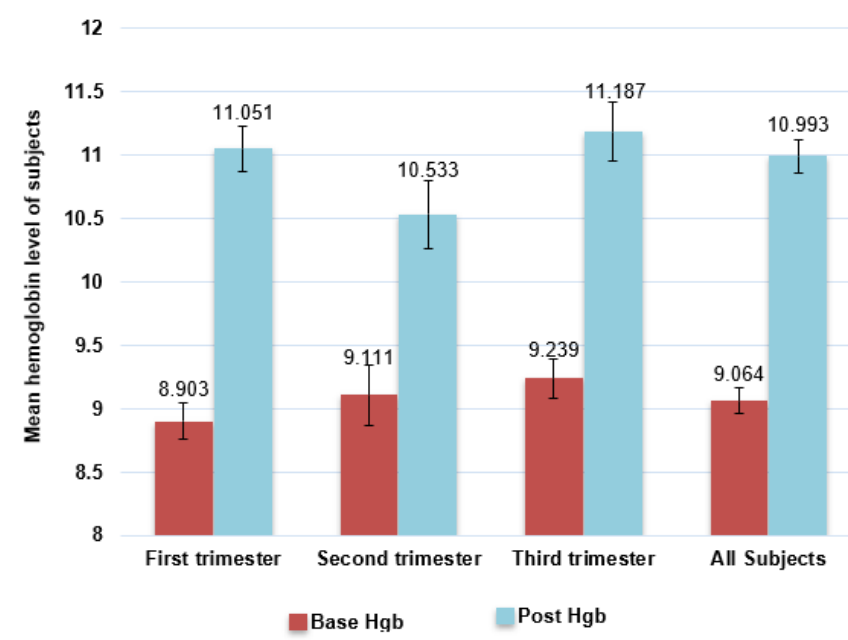

Figure 2. This figure showed the discrepancy of the first, second and third trimester before and after FMC compared with the total average before and after FMC

hemoglobin than mild and/or moderate cases after a single dose (1000 $\mathrm{mg}$ ) of FCM. All cases related to severity of IDA had been shifted from severe, moderate, mild to moderate, mild, healthy respectively. Only one case with severe IDA remained severe after using FCM, therefore another dose maybe warranted. 
Treatment of IDA will never lead to iron toxicity, however, in case of injection of higher dose above the requirements, it needs further monitoring although teratogenicity is with limited possibility, specifically if the treatment within the first trimester. If iron accumulation has been noticed, intravenous deferoxamine should be administered as chelation therapy [27]. Excessive Hgb levels were not observed in this study, all participants on FCM injection had elevated levels that fell within normal ranges.

Traditionally, blood transfusion is considered as an option in severe anemic cases; especially when oral replacement is not effectively and rapidly replenished the iron stores. The newly developed iron formulation such as FCM is offering the benefit of administrating a single higher dose with rapid, effective and safe repletion of iron, which can employ to avoid the risk of blood transfusion in severe anemia.

This study could not find any correlation between Hgb levels and education, job, number of pregnancies, the consumed iron from food (vegetarian or not), and pagophagia.

A side issue related to the cost of treatment in comparison between FCM and oral ferrous treatment considering that the cost of FCM is $200 \$$ per injection of $1000 \mathrm{mg}$ single dose with almost absolute treatment for more than 6 months and only one visit comparing with multiple visits, cost more than 200\$ USD per each visit, and irregular treatment subjecting premature births and low birth weight babies and delayed growth and development.

\section{Conclusion}

This study approved that one intravenous injection of FCM improves the hemoglobin level in a significant consideration in anemic pregnant women. Also, it showed that the respond of severe anemic cases were more positive in improving the level of hemoglobin than mild and/or moderate cases. Only one case remained severe despite receiving one dose of FCM. Dizziness was experienced and reported by only one pregnant woman at the emergency department post FCM injection, which indicated its effectiveness and safety. Blood transfusion could be avoided by using FCM injection to optimize the iron stores rapidly and effectively. The proposed recommendation is that intravenous injection of FCM could be used as trend in the first and second trimester during pregnancy with or without IDA if the hemoglobin level is low. Moreover, intravenous iron can be given late in pregnancy in the third trimester when rapid restoration of the iron stores and hemoglobin is required to avoid blood transfusion at delivery, it is also often needed after delivery when there is excessive bleeding in labor.

\section{Acknowledgment}

We would like to express our deepest appreciation to the health sciences research center. A special gratitude to all co-workers in the gynaecology clinics at King Abdullah University Hospital and those who gave us the permission to use all the required access to complete the study. Furthermore, we are indebted to all pregnant women involved in this study.

\section{Conflict of interest}

The authors reported no potential conflict of interest.

\section{References}

1. Idris M, Anis-ur-Rehman (2005) Iron deficiency anaemia in moderate to severely anaemic patients. J Ayub Med Coll Abbottabad 17: 45-47. [Crossref]
2. McLean E, Cogswell M, Egli I, Wojdyla D, de Benoist B (2009) Worldwide prevalence of anaemia, WHO vitamin and mineral nutrition information system, 1993-2005. Public Health Nutr 12: 444-454. [Crossref]

3. Bakhtiar UB, Khan Y, Nasar R (2007) Relationship between maternal hemoglobin and perinatal outcome. Age (years) 25: 24.

4. Siti-Noor AS, Wan-Maziah WM, Narazah MY, Quah BS (2006) Prevalence and risk factors for iron deficiency in Kelantanese pre-school children. Singapore Med J 47: 935-939. [Crossref]

5. Jackson RT, Al-Mousa Z (2000) Iron deficiency is a more important cause of anemia than hemoglobinopathies in Kuwaiti adolescent girls. J Nutr 130: 1212-1216. [Crossref]

6. Abbaspour N, Hurrell R, Kelishadi R (2014) Review on iron and its importance for human health. J Res Med Sci 19: 164-174. [Crossref]

7. Pawlak R, Berger J, Hines I (2018) Iron Status of Vegetarian Adults: A Review of Literature. Am J Lifestyle Med 12: 486-498. [Crossref]

8. AlQuaiz JM, Abdulghani HM, Khawaja RA, Shaffi-Ahamed S (2012) Accuracy of various iron parameters in the prediction of iron deficiency anemia among healthy women of child bearing age, Saudi Arabia. Iran Red Crescent Med J 14: 397-401. [Crossref]

9. Alem M, Enawgaw B, Gelaw A, Kenaw T, Seid M et al. (2013) Prevalence of anemia and associated risk factors among pregnant women attending antenatal care in Azezo Health Center Gondar town, Northwest Ethiopia. J Interdiscipl Histopathol 1: 137-144.

10. Khalafallah AA, Dennis AE (2012) Iron deficiency anaemia in pregnancy and postpartum: pathophysiology and effect of oral versus intravenous iron therapy. $J$ Pregnancy. [Crossref]

11. Bhatia MS, Kaur N (2014) Pagophagia A common but rarely reported form of pica. $J$ Clin Diagn Res 8: 195-196. [Crossref]

12. Di Renzo GC, Spano F, Giardina I, Brillo E, Clerici G, et al. (2015) Iron deficiency anemia in pregnancy. Women's Health 11: 891-900.

13. Scholl TO (2011) Maternal iron status: relation to fetal growth, length of gestation, and iron endowment of the neonate. Nutr Rev 69: S23-S29. [Crossref]

14. Radlowski EC, Johnson RW (2013) Perinatal iron deficiency and neurocognitive development. Front Hum Neurosci 7: 585. [Crossref]

15. Froessler B, Collingwood J, Hodyl NA, Dekker G (2014) Intravenous ferric carboxymaltose for anaemia in pregnancy. BMC pregnancy childbirth 14: 115 . [Crossref]

16. Pels A, Ganzevoort W (2015) Safety and efficacy of ferric carboxymaltose in anemic pregnant women: a retrospective case control study. Obstet Gynecol Int 2015:728952. [Crossref]

17. Breymann C, Gliga F, Bejenariu C, Strizhova N (2008) Comparative efficacy and safety of intravenous ferric carboxymaltose in the treatment of postpartum iron deficiency anemia. Int J Gynaecol Obstet 101: 67-73. [Crossref]

18. Froessler B, Cocchiaro C, Saadat-Gilani K, Hodyl N, Dekker G (2013) Intravenous iron sucrose versus oral iron ferrous sulfate for antenatal and postpartum iron deficiency anemia: a randomized trial. J Matern Fetal Neonatal Med 26: 654-659. [Crossref]

19. Gupta A, Avanthi G (2018) Iron Deficiency Anaemia in Pregnancy: Developed Versus Developing Countries. EMJ Hematol 6: 101-109.

20. Centers for Disease Control and Prevention (1998) Recommendations to Prevent and Control Iron Deficiency in the United States. MMWR Recomm Rep 47: 1-36.

21. WHO (2011) Haemoglobin concentrations for the diagnosis of anaemia and assessment of severity.

22. Nutrition Working Group, O'Connor DL, Blake J, Bell R, Bowen A, et al. (2016) Canadian consensus on female nutrition: adolescence, reproduction, menopause, and beyond. J Obstet Gynaecol Can 38: 508-554. [Crossref]

23. Seeho SKM, Morris JM (2018) Intravenous iron use in pregnancy: Ironing out the issues and evidence. Aust N Z J Obstet Gynaecol 58: 145-147. [Crossref]

24. Aporta Rodriguez R, García Montero M, Lorente Aporta JP, Gallego Luque C, Chacón Mayor A, et al. (2016) Retrospective case reports of anemic pregnant women receiving intravenous ferric carboxymaltose: Experience from a tertiary hospital in Spain. Obste Gynecol Int 2016: 5060252. [Crossref] 
Alyami A (2019) The effectiveness of ferric carboxymaltose in childbearing age with iron deficiency anemia

25. Froessler B, Gajic T, Dekker G, Hodyl NA (2018) Treatment of iron deficiency and iron deficiency anemia with intravenous ferric carboxymaltose in pregnancy. Arch Gynecol Obstet 298: 75-82. [Crossref]
26. D Zeba, PA Khanam, M Ahmed, MA Khair (2017) Intravenous Iron Treatment in Pregnancy: Ferric Carboxymaltose for Correction of Iron Deficiency Anaemia. Faridpur Med Coll 12: 54-57.

27. Fine JS (2000) Iron poisoning. Curr Prob Pediatr 30: 71-90

Copyright: (2019 Alyami A. This is an open-access article distributed under the terms of the Creative Commons Attribution License, which permits unrestricted use, distribution, and reproduction in any medium, provided the original author and source are credited. 